Research Paper

\title{
Extensively disturbance of regulatory T cells - Th17 cells balance in stage II pulmonary sarcoidosis
}

\author{
Jingjing Ding1, Jinghong Dai ${ }^{1}$, Hourong Cai ${ }^{1}$, Qian Gao ${ }^{2 \bowtie}$, Yanting Wen ${ }^{2 \bowtie}$ \\ 1. Department of Respiratory Medicine, Jiangsu Key Laboratory of Molecular Medicine, the Affiliated Drum Tower Hospital of Nanjing University Medical \\ School, Nanjing, China \\ 2. Center of Translational Medicine, Jiangsu Key Laboratory of Molecular Medicine, Nanjing University Medical School, Nanjing, China
}

$\triangle$ Corresponding authors: Yanting Wen, Email: wenyanting@nju.edu.cn. Qian Gao, Email: qian_gao@nju.edu.cn. Center of Translational Medicine, Jiangsu Key Laboratory of Molecular Medicine, Nanjing University Medical School, China. Address: Center of Translational Medicine, Nanjing University Medical School, 22 Hankou Road, Nanjing 210093, China. Tel: 86-25-83594757; Fax: 86-25-83593376.

(c) Ivyspring International Publisher. This is an open access article distributed under the terms of the Creative Commons Attribution (CC BY-NC) license (https://creativecommons.org/licenses/by-nc/4.0/). See http://ivyspring.com/terms for full terms and conditions.

Received: 2016.12.20; Accepted: 2017.07.05; Published: 2017.09.04

\begin{abstract}
Background: Sarcoidosis is a systemic inflammatory disorder characterized by granulomas. Not enough evidences correlate the derangement of CD4+ T subsets, which have an impact on the therapeutic effects of corticosteroids, with the radiographical staging of sarcoidosis. Here we show the disturbance of CD4+ T subsets in newly diagnosed stage II pulmonary sarcoidosis, which is the most common stage in which corticosteroids treatment is used.

Materials and methods: 39 newly diagnosed and treatment-naïve patients and 9 subjects after corticosteroids treatment were included. CD4+ CD45RA+/ CD45RO+ cells, CCR4+ CCR6+ cells, and $T$ regulatory cells (Tregs) were tested by Flow Cytometry Analysis. Th1/Th2, Tregs/Th17 related cytokines and mRNAs, SAA and CCL20 were also measured. The activation of PI3K/PTEN/Akt signaling pathway was detected.

Results: Percentages of CD4+CD45RO+ memory T cells and Tregs, serum levels of IL-17A, TGF- $\beta 1$, IL-6, IFN- $\gamma$, IL-10, SAA and CCL20, copies of T-bet, FoxP3, IL-17 and RORc in the periphery were elevated in newly diagnosed stage II pulmonary sarcoidosis patients. Additionally, PI3K/Akt signaling pathway was activated in bronchoalveolar lavage fluid cells.

Conclusions: Disturbance of T memory cells, Th1/Th2, and Tregs/Th17 cells, and activation of PI3K/Akt signaling were seen in newly diagnosed stage II pulmonary sarcoidosis, which can be partly ameliorated by corticosteroids treatment.
\end{abstract}

Key words: Stage II pulmonary sarcoidosis; Th17 cells; Tregs; PI3K/Akt signaling pathway

\section{Introduction}

Sarcoidosis is a multisystem chronic condition of unknown etiology, characterized by the formation of immune granulomas in various organs, mainly the intrathoracic lymph nodes and the lungs, although any organ can be involved $[1,2]$. Chest radiographs in patients with sarcoidosis have been classified into four stages: stage I (lymphadenopathy alone), stage II (pulmonary infiltration with lymphadenopathy), stage III (pulmonary infiltration without lymphadenopathy), and stage IV (pulmonary fibrosis) [1]. To palliate symptoms, to switch off acute inflammation, and to prevent end-organ disease, treatment strategies mainly rely on immunesuppression with steroids and/or steroid-sparing drugs [3].

Different subsets of CD4+ helper $\mathrm{T}$ cells participate in the immunopathogenesis of sarcoidosis. The main feature of the acute disease was represented by a Th1/Th17/regulatory $\mathrm{T}$ cells (Tregs) -driven inflammatory process, involving macrophages both as antigen-presenting cells and key effectors [3-5]. However, not enough evidences correlate the derangement of CD4+ $\mathrm{T}$ subsets with the radiographical staging of sarcoidosis. A randomized, 
placebo controlled, double-blind, parallel-group, multicenter studies demonstrated that immediate treatment of pulmonary stage II sarcoidosis with corticosteroids, but not stage I disease, improved the 5 -year prognosis with regard to lung function variables [6,7]. Corticosteroids treatment influence the immunopathogenesis of sarcoidosis, by modulating a number of genes, which are expressed soon after antigen triggering of $\mathrm{CD} 4+\mathrm{T}$ cells [8]. As a result, diversity of $\mathrm{CD} 4+\mathrm{T}$ cells' disturbance among different stages of sarcoidosis may have an impact on the therapeutic effects of corticosteroids. The aim of this study is to show the disturbance of CD4+ T cells in in stage II pulmonary sarcoidosis, which is the most common disease stage in which corticosteroids treatment is used.

\section{Materials and methods}

\section{Patient characteristics}

Patients, whose stage II sarcoidosis was a diagnostic consideration or was after GC treatment, were prospectively enrolled from in- or out-patients of Department of Respiratory Medicine, the Affiliated Drum Tower Hospital of Nanjing University Medical School. Bronchoalveolar lavage fluid (BALF) samples were obtained from the diagnostic bronchoscopy if there were extra samples besides pathology tests, while peripheral blood samples were obtained before the initial diagnosis from those newly diagnosed patients or during the follow-up. To define sarcoidosis, the clinical, histological, and microbiologic criteria were used as previously described [9]. Radiographic staging was performed and 44 stage II sarcoidosis patients (16 men and 28 women; mean age $51.8 \pm 1.53$ years) were finally enrolled in assay. In these 44 patients enrolled, 39 were newly diagnosed and treatment-naive, the other 5 have been administered GC for a period from 1 month to 1 year. Blood samples from 4 of the 39 newly diagnosed sarcoidosis patients were both collected before initial diagnosis and during the follow-up 1 to 3 months after GC treatment. This study was performed in conformity with the Declaration of Helsinki of the World Medical Association and approved by the Ethics Committee of the Affiliated Drum Tower Hospital of Nanjing University Medical School. All subjects provided written informed consent prior to their enrollment. The control group consisted of 44 otherwise healthy individuals (16 men and 28 women; mean age $50.0 \pm 1.28$ years), who were recruited from the physical examinees in the Affiliated Drum Tower Hospital of Nanjing University Medical School.

\section{Flow Cytometry Analysis (FACS) of Intracellular Cytokines}

Peripheral blood mononuclear cells (PBMCs) were freshly isolated. To analyze intracellular cytokines, FACS was used to detect the CD25 + FoxP3+ cells by gating CD4+ cells, CCR4+ CCR6+ cells, CD4+ CD45RA+ cells and CD4+CD45RO+ cells in PBSCs using a FACSCalibur system (BD Biosciences, San Jose, CA). FITC conjugated CD4, APC conjugated CD25, PE conjugated Foxp3, PE conjugated CCR4, APC conjugated CCR6, PE conjugated CD45RA, APC conjugated CD45RO, and appropriate isotype controls were purchased from eBioscience (Miltenyi Biotec, CA, USA).

\section{Real-Time Quantitative Polymerase Chain Reaction (PCR)}

The mRNA expression levels were estimated using a StepOnePlus ${ }^{\mathrm{TM}}$ Real-Time PCR System (Applied Biosystems, USA). All reactions were performed with the power SYBR Green PCR Master Mix (Applied Biosystems, USA), following the manufacturer's instructions. The mRNA expression was normalized to that of GAPDH. PCR procedures were as follows: $95{ }^{\circ} \mathrm{C}$ for $10 \mathrm{~min}$ followed by 40 cycles at $95^{\circ} \mathrm{C}$ for $15 \mathrm{~s}, 60{ }^{\circ} \mathrm{C}$ for $60 \mathrm{~s}, 72{ }^{\circ} \mathrm{C}$ for $10 \mathrm{~s}$. Each sample was analyzed in triplicate. The relative amount of each target was expressed as $2^{-} \Delta \Delta \mathrm{CT}$ values. The primers sequences are shown in supplementary Table.

\section{Multiple cytokine assays}

Serum samples were centrifuged at $1500 \mathrm{rpm}$ for $10 \mathrm{~min}$ to remove cell debris. IL-4, IL-6, IL-10, IL-17A, IL-17E, IL-17F, CCL20, and IFN- $\gamma$ were measured using MILLIPLEX MAP Human TH17 Magnetic Bead Panel (MILLIPLEX, MA, USA) per manufacturer instructions. Soluble TGF- $\beta 1$ and serum amyloid A (SAA) levels were assayed using a commercial ELISA kit (CUSABIO, Wuhan, China).

\section{Bronchoalveolar lavage collection and western blot}

Bronchoscopy with BAL samples were centrifuged at $1500 \mathrm{rpm}$ for $10 \mathrm{~min}$ to separate BALF from the cell component, and then were lysed in RIPA lysis buffer (Beyotime, Haimen, China) using a homogenizer on ice. Western blot analysis was performed in 4 BAL cell samples from stage II sarcoidosis patients and 4 as controls from those who were submitted to bronchoscopy for other reasons, mainly chronic cough and hemoptysis. No subjects in the control group suffered from malignant, inflammatory, or interstitial lung disease. Mouse anti-mouse Akt antibody, rabbit anti-mouse pAkt 
(S473) antibody, mouse anti-mouse PTEN antibody, and mouse anti-mouse PI3K antibody (1:1000) (R\&D, MN, USA) were used for the detection of proteins. Determined by densitometry, the level of each protein was normalized to GAPDH.

\section{Statistical Analysis}

All data were expressed as the mean \pm standard error (SE). Statistical analysis was performed using SPSS 18.0 software (SPSS Inc., Chicago, IL). Comparing numerical data between groups was performed with One-way ANOVA. P values $<0.05$ were considered statistically significant.

\section{Results}

\section{Naïve/memory $T$ cells}

Although the percentage of CD4+ cells and CD4+CD45RA+ cells demonstrated no statistical change among groups, $\mathrm{CD} 4+\mathrm{CD} 45 \mathrm{RO}+$ cells in peripheral blood were significantly higher in patients with sarcoidosis compared to control subjects (34.1 \pm $5.0 \%$ vs $24.1 \pm 1.6 \%, \mathrm{P}=0.39$ ), while showing no statistical differences after glucocorticoid (GC) treatment comparing to group sarcoidosis and healthy controls (Fig. 1A, B and C).

\section{Tregs and Th17 subsets}

As shown in Fig. 1D, CD25+FoxP3+ Tregs greatly increased in stage II sarcoidosis patients in comparison with healthy subjects $(35.1 \pm 3.9 \%$ vs 13.3 $\pm 0.9 \%, \mathrm{P}=0.000$ ). An improvement of Tregs' level was seen in sarcoidosis patients after GC treatment (25.4 $\pm 8.1 \%)$, showing no statistical difference comparing to healthy controls $(\mathrm{P}=0.082)$. The expression of the mRNA for the Tregs-specific transcription factor FoxP3 was relatively higher in sarcoidosis patients compared with that in healthy controls $(P=0.003)$, while GC treatment eliminated this increase (Fig. 2A).

As surface markers for CD4+ Th17 cell subsets [10], CCR4 and CCR6 co-expression (Fig 1E) was not statistically changed among groups control, sarcoidosis and GC treatment. With regard to Th17 cytokines, there was an increased expression of IL-17A in sarcoidosis patients in comparison with healthy controls $(127.5 \pm 10.1 \mathrm{ng} / \mathrm{ml}$ vs $83.9 \pm 9.0$ $\mathrm{ng} / \mathrm{ml}, \mathrm{P}=0.005)$, and no statistical differences between groups GC treatment and healthy controls (Fig. 3A). No statistical differences were seen in IL-17F among groups (Fig. 3B). The levels of TGF- $\beta 1$ and IL-6 were both increased in peripheral blood in sarcoidosis patients compared with controls $(0.42 \pm 0.03 \mathrm{ng} / \mathrm{ml}$ vs $0.29 \pm 0.02 \mathrm{ng} / \mathrm{ml}, 8.0 \pm 5.6 \mathrm{ng} / \mathrm{ml}$ vs $19.7 \pm 2.8 \mathrm{ng} / \mathrm{ml}$, $\mathrm{P}=0.018$ and $\mathrm{P}=0.001$, respectively); after $\mathrm{GC}$ treatment, IL-6 was still elevated $(47.9 \pm 16.3 \mathrm{ng} / \mathrm{ml}, \mathrm{P}$ $=0.015)$, while there was no differences of TGF- $\beta 1$ compared with controls (Fig.3C, D). Compared with healthy controls, sarcoidosis patients had a significantly increased copies of the IL-17 and RORc mRNA ( $\mathrm{P}=0.005$ and $\mathrm{P}=0.013$, respectively), but normal IL-17 and RORc expressions after GC treatment (Fig. 2B, C). The levels of CCL20 were more pronounced in sarcoidosis than in control $(129.3 \pm 11.3$ $\mathrm{ng} / \mathrm{ml}$ vs $88.6 \pm 8.1 \mathrm{ng} / \mathrm{ml}, \mathrm{P}=0.004)$, and still had a trend of increasing after GC treatment (129.9 \pm 20.1 ng/ml, $\mathrm{P}=0.057$ ) (Fig. 3J).
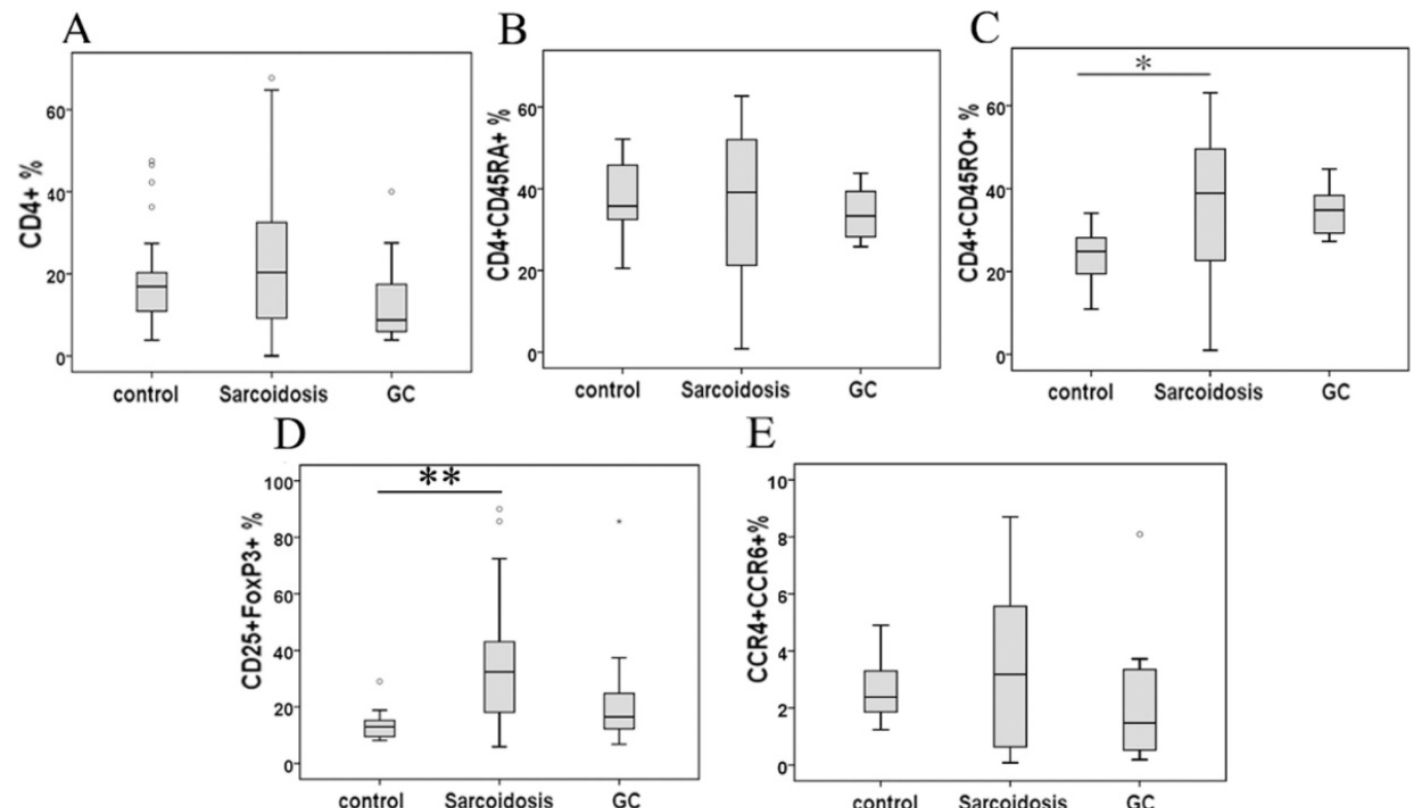

E

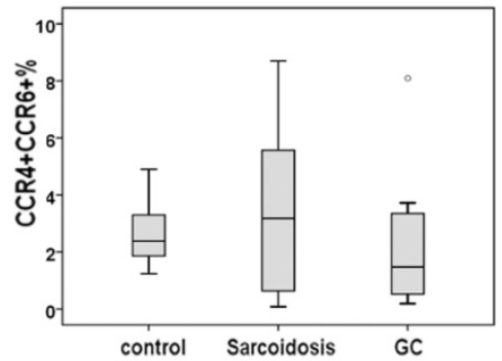

FIG. 1. Percentage of CD4+ lymphocytes (A), CD4+ CD45RA+ cells (B), CD4+ CD45RO+ cells (C), CD25+FoxP3+ cells (D), and CCR4+ CCR6+ cells in CD4+ cells $(E)$ in peripheral blood mononuclear cells analyzed by FACS. *Difference was significant compared to normal controls $(P<0.05)$. **Difference was significant compared with normal controls $(P<$ 0.01 ). 

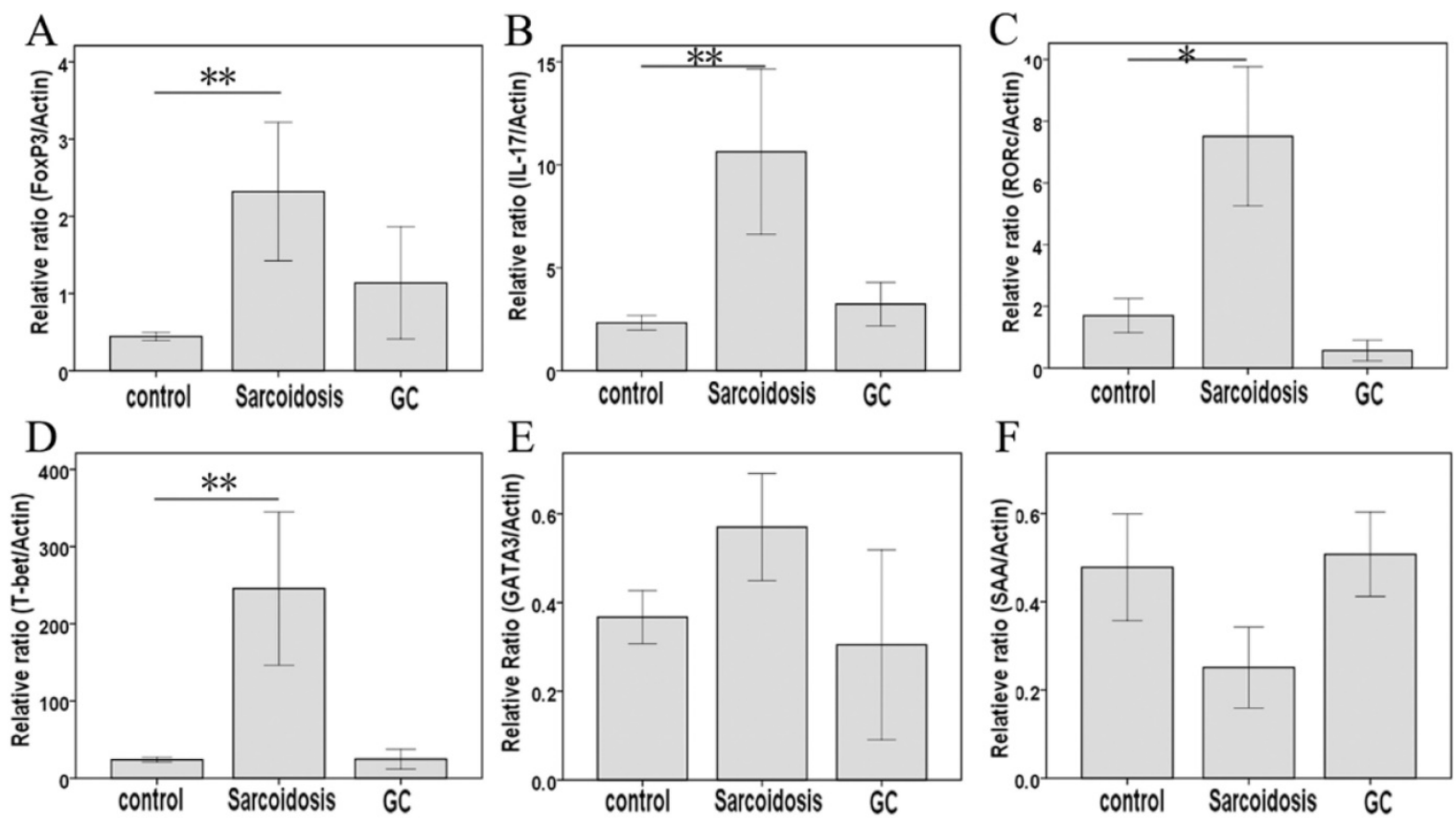

FIG. 2. FoxP3, IL-17, RORc, T-bet, GATA3, and SAA mRNA measured by means of quantitative real-time PCR. *Difference was significant compared with normal controls $(P<0.05)$; **Difference was significant compared with normal controls $(P<0.01)$.
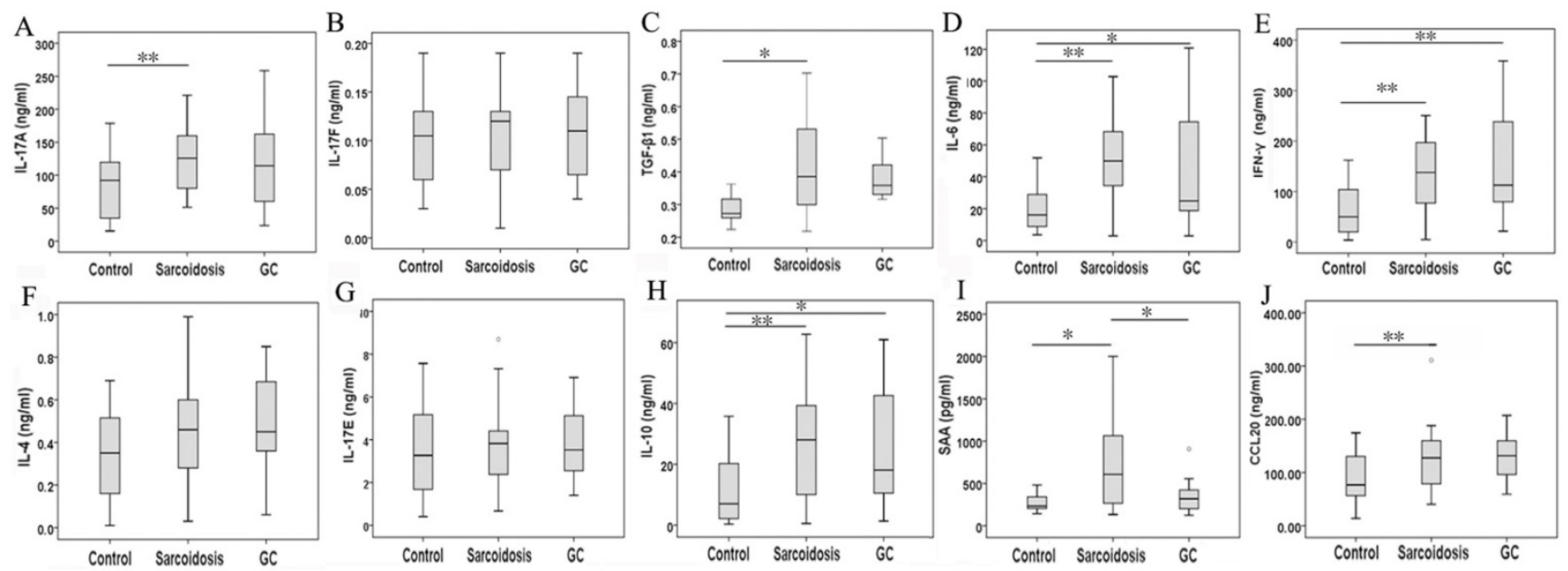

FIG. 3. Serum IL-17A, IL-17F, TGF- $\beta 1$, IL-6, IFN- $\gamma$, IL-4, IL-17E, IL-10, SAA, and CCL20 levels determined by MILLIPLEX MAP Human TH17 Magnetic Bead Panel or by ELISA. *Difference was significant $(P<0.05)$. **Difference was significant $(P<0.01)$.

\section{Th1 and Th2 subsets}

In serum, Th1 cytokine such as IFN- $\gamma$ was elevated in groups sarcoidosis and GC treatment compared with group healthy control $(161.2 \pm 45.4$ $\mathrm{ng} / \mathrm{ml}, 140.7 \pm 13.3 \mathrm{ng} / \mathrm{ml}$ vs $63.5 \pm 8.9 \mathrm{ng} / \mathrm{ml}, \mathrm{P}=$ 0.000 and $\mathrm{P}=0.001$, respectively), but did not differ between groups sarcoidosis and GC treatment (Fig. 3E). Meanwhile, the expression of T-bet mRNA was elevated $(P=0.004)$ in sarcoidosis patients, and normalized after GC treatment. The levels of SAA was increased in sarcoidosis in comparison with control $(710.3 \pm 120.0 \mathrm{pg} / \mathrm{ml}$ vs. $278.4 \pm 44.8 \mathrm{pg} / \mathrm{ml}, \mathrm{P}=0.021)$. Although there was no statistical differences of SAA
mRNA copies among groups (Fig. 2F), GC treatment ameliorated SAA level to normal $(364.1 \pm 81.7 \mathrm{pg} / \mathrm{ml}$, $P=0.647$ ) in serum (Fig. 3I).

With regard to Th2 cytokines, both IL-4 and IL-17E/IL25 showed no significant differences among groups sarcoidosis, control and GC treatment (Fig. 3F, G). The copies of GATA3 mRNA demonstrated no changes in sarcoidosis and after treatment (Fig. 2D, E). However, IL-10 was significantly elevated in groups sarcoidosis, and GC treatment while comparing to healthy control $(26.5 \pm 3.2 \mathrm{ng} / \mathrm{ml}, 26.7 \pm 8.4 \mathrm{ng} / \mathrm{ml}$ vs $11.0 \pm 1.9 \mathrm{ng} / \mathrm{ml}, \mathrm{P}=0.000$ and $\mathrm{P}=0.014$, respectively) (Fig. $3 \mathrm{H})$. 


\section{Activated PI3K/PTEN/Akt pathway in sarcoidosis}

To investigate whether defects in PI3K/PTEN/Akt signaling pathway were associated with the pathogenesis of sarcoidosis in patients, whole protein lysates of bronchoalveolar lavage (BAL) cells isolated from sarcoid patients or controls were immunoblotted. We found that Akt and pAkt protein levels were significantly elevated in sarcoidosis $(\mathrm{P}=0.023$ and $\mathrm{P}=0.015$, respectively) (Fig.4). The levels of PI3K protein also had a trend of increasing, although did not reach statistical difference $(P=0.06)$. In contrast, levels of the PTEN protein in these BAL cells were equivalent to levels found in normal controls. These results suggest a specific activation of PI3K/Akt pathway in BAL cells in sarcoidosis patients.

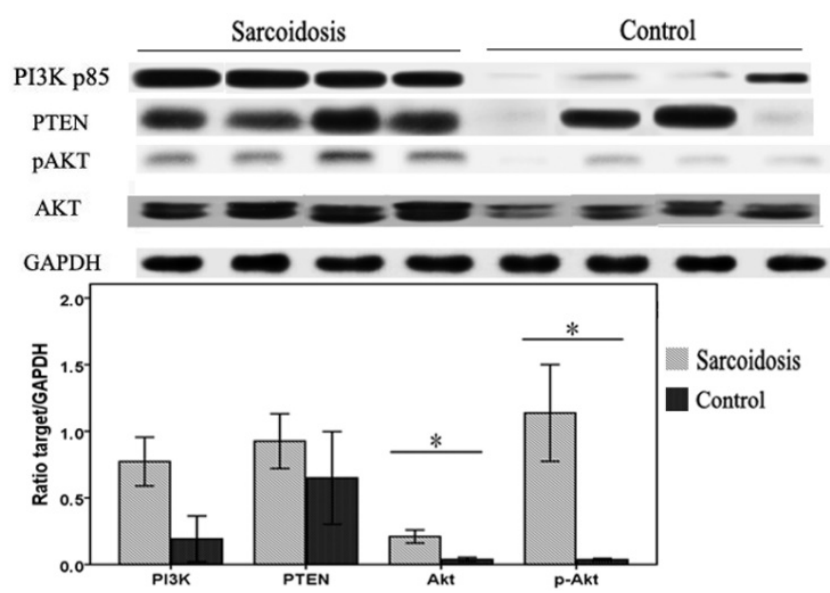

FIG. 4. Bronchoalveolar lavage $(B A L)$ cells were subjected to immunoblot analysis with antibodies against PI3K, PTEN, Akt, pAkt, and GAPDH. Top: Representative immunoblots of PI3K, PTEN, Akt, pAkt, and GAPDH protein expression in total protein extracts from the BAL cells. Bottom: Results of densitometry analyses were reported. *Difference was significant $(P<0.05)$.

\section{Discussions}

In this study, we found elevated levels of CD4+CD45RO+ memory $\mathrm{T}$ cells and Tregs in patients with stage II pulmonary sarcoidosis. Although the percentage of CCR4+CCR6+ cells did not change in stage II pulmonary sarcoidosis, serum levels of IL-17A, TGF- $\beta 1$ and IL-6 were increased. Together with the disturbance of Tregs/Th17 cells balance, PI3K/Akt signaling pathway was found to be activated. Circulating Th1 cytokine such as IFN- $\gamma$ and Th2 cytokine such as IL-10 was elevated, while both IL-4 and IL-17E/IL25 did not differ among groups. As a component of the innate immune response regulating granulomatous inflammation in sarcoidosis through Toll-like receptor-2, SAA, which influence Th1/Th2 balance and granuloma formation
[11], was more pronounced in recently diagnosed stage II pulmonary sarcoidosis, and was normalized after GC treatment.

Sarcoidosis bears the hallmarks of a Th1/Treg/Th17-mediated disease in which activated $\mathrm{T}$ cells and cytokines are associated with pathogenesis $[12,13]$. In patients with sarcoidosis, the pulmonary microenvironment is characterized by a well-known highly polarized Th1 profile. Local imbalance of Th1/Th2 with the shift towards Th1-related cytokine expression, with the predominance of Th1-related cytokines (e.g., IL-2, IFN- $\gamma$, and TNF-a) over Th2-related cytokines (e.g., IL-4, IL-5, and IL-10) is observed in the BALF [14], associated with the high expression of macrophage-derived molecules such as IL-15, CXCL10, CXCL16, CCL5 and CCL20 [5]. IL-17-producing Th17 cells and increased levels of IL-17A participate in the alveolitic/granuloma phase, to the maintenance of granuloma and also to the progression towards the fibrotic phase of the disease $[5,15,16]$. The recruitment of Th17 cells to granulomas is due to the release of cytokines and chemokines such as IL-1 $\beta$, IL-6, IL-23, IL-12, IL-18, and CCL20 by locally and systemically activated macrophages [16]. Expressing a specific master transcription factor known as RORc, human Th17 cells comprise heterogeneous subsets. Next to IL-17A, these cells produce various other proinflammatory cytokines, including IL-17F, IL-22 and, in some conditions, IFN- $\gamma$ [15]. Hereby we demonstrated normal levels of CCR4+CCR6+ cells, but enhanced serum IL-17A expression and increased IL-17 and RORc mRNA in recently diagnosed stage II sarcoidosis patients. Meanwhile, CCL20, which is the only chemokine produced and released by Th17 cells [5], was also overexpressed in those patients. GC treatment neutralized serum IL-17A, as well as IL-17 and RORc mRNA in PBMC, suggesting neutralization of IL-17A and RORc may be a novel strategy to treat stage II sarcoidosis.

The concomitant presence of the intense immune response in the affected organs and a suppressed antigen immune response in the periphery was deeply affected by Tregs of patients with sarcoidosis. While an unlimited inflammatory response suggests an impaired immune control in sarcoid lesions, it stands in contrast to the massive infiltration with Tregs expanding both peripherally and intra-tissularly. Although there are healthy Tregs which suppress the initial steps of granuloma formation, these cells have no positive influence on sarcoidosis lesions, displaying poor suppressive capacity [17-19]. In consistence with previous reports, it is demonstrated by our data that Tregs and FoxP3 copies were more prevalent in patients with newly 
diagnosed stage II sarcoidosis than controls, and both Tregs and FoxP3 copies were normalized after GC treatment. Tregs in sarcoidosis exhibited reduced repressor capacities despite high IL-10 and TGF- $\beta 1$ levels [18]. We here revealed that after GC treatment, the levels of TGF- $\beta 1$ but not IL-10 was normalized, which might help the reversal of Tregs dysfunction.

TGF- $\beta 1$ is critical for commitment to the development of both Tregs and Th17 subsets, which decides the anti-inflammatory (Tregs) or pro-inflammatory (Th17) effects. With the production of TGF- $\beta$, Tregs mediates immune-responsesuppressive functions. Meanwhile, by acting together with IL-6, TGF- $\beta 1$ induces the development of Th17 cells and inhibits Tregs differentiation [20-23]. The more pronounced proinflammatory IL-6, TGF- $\beta 1$ may help convert Tregs into Th17 lymphocytes in newly diagnosed stage II sarcoidosis patients. TGF- $\beta 1$ levels were more pronounced in Sarcoidosis comparing to normal controls. After GC treatment, its level had no difference with group control, although have no statistical difference with group Sarcoidosis either. In this context, GC treatment might somehow help neutralizing TGF- $\beta 1$, might restrain Th17 development and control the inhibition of Tregs differentiation. Although pooled data from our study did not support the restrain potential of Th17 or CCL20 after GC treatment, based on the limited patients' numbers in group GC, future studies on Th17 are still warranted.

PI3K/Akt/mTOR signaling network regulates Foxp3 expression and inhibits the peripheral induction of Tregs [24]. In contrast, pharmacological inhibition of PI3K signaling enhances Tregs differentiation in vitro [24]. As a strong repressor of entry into the Tregs phenotype in vitro and in vivo, activation of Akt leads to an overall dampening of the Tregs gene signature, including reduced expression of Foxp3, Il2ra and Ctla4 [25]. Sustained activation of Akt not only influences the induction of Tregs, but also restrains the immune suppressive effects played by Tregs [24, 26, 27]. Here we report activated PI3K/Akt signaling pathway in BAL cells in newly diagnosed stage II sarcoidosis. These results place PI3K/Akt at a nexus of signaling pathways whose activation may impact on the impaired function of Tregs in sarcoidosis. In fact, controlling of PI3K signaling in Tregs is proven critical for maintaining their homeostasis, function and stability both in vitro and in vivo $[28,29]$. Controlling PI3K/Akt signaling pathway may be harnessed for therapeutic benefit in stage II sarcoidosis.

In conclusion, our data highlight the presence of Th1/Th2 and Th17/Tregs imbalance in periphery and the activation PI3K/Akt pathway in the BAL cells of patients with newly diagnosed stage II sarcoidosis. Our data favor the hypothesis that the Tregs malfunction in sarcoidosis may be partly driven by the activation of PI3K/Akt signaling pathway. Further studies are needed to better understand the role of PI3K/PTEN/Akt signaling pathway in both the various disease stages and the treatment responses of stage II sarcoidosis and to validate the possibility of a treatment based on Th17/Tregs and/or PI3K/Akt neutralization.

\section{Acknowledgments}

This work was supported by the National Natural Science Foundation of China (81100386, 81400046), the Independent and Open Grant of Jiangsu Key Laboratory of Molecular Medicine.

\section{Author Contributions}

J.D. Y.W. and Q.G. conceived and designed the experiments, and wrote the paper; J.D., JH.D. Y.W. and H.C. performed the experiments and analyzed the data.

\section{Competing Interests}

The authors have declared that no competing interest exists.

\section{References}

1. Valeyre D, Prasse A, Nunes H, et al. Sarcoidosis. Lancet. 2014; 383: 1155-67.

2. Iannuzzi MC, Rybicki BA, Teirstein AS. Sarcoidosis. N Engl J Med. 2007; 357: 2153-65.

3. Cinetto F, Agostini C. Advances in understanding the immunopathology of sarcoidosis and implications on therapy. Expert Rev Clin Immunol. 2016; 12: 973-88.

4. Urbankowski T, Hoser G, Domagala-Kulawik J. Th1/Th2/Th17related cytokines in the bronchoalveolar lavage fluid of patients with sarcoidosis: association with smoking. Pol Arch Med Wewn. 2012; 122: 320-5.

5. Facco M, Cabrelle A, Teramo A, et al. Sarcoidosis is a Th1/Th17 multisystem disorder. Thorax. 2011; 66: 144-50.

6. Pietinalho A, Tukiainen P, Haahtela T, et al. Oral prednisolone followed by inhaled budesonide in newly diagnosed pulmonary sarcoidosis: a double-blind, placebo-controlled multicenter study. Finnish Pulmonary Sarcoidosis Study Group. Chest. 1999; 116: 424-31.

7. Pietinalho A, Tukiainen P, Haahtela T, et al. Early treatment of stage II sarcoidosis improves 5-year pulmonary function. Chest. 2002; 121: 24-31.

8. Munck A, Mendel DB, Smith LI, et al. Glucocorticoid receptors and actions. Am Rev Respir Dis. 1990; 141: S2-10.

9. Hunninghake GW, Costabel U, Ando M, et al. ATS/ERS/WASOG statement on sarcoidosis. American Thoracic Society/European Respiratory Society/World Association of Sarcoidosis and other Granulomatous Disorders. Sarcoidosis Vasc Diffuse Lung Dis. 1999; 16: 149-73.

10. Acosta-Rodriguez EV, Rivino L, Geginat J, et al. Surface phenotype and antigenic specificity of human interleukin 17-producing $\mathrm{T}$ helper memory cells. Nat Immunol. 2007; 8: 639-46.

11. Chen ES, Song Z, Willett $\mathrm{MH}$, et al. Serum amyloid A regulates granulomatous inflammation in sarcoidosis through Toll-like receptor-2. Am J Respir Crit Care Med. 2010; 181: 360-73.

12. Georas SN, Chapman TJ, Crouser ED. Sarcoidosis and T-Helper Cells. Th1, Th17, or Th17.1? Am J Respir Crit Care Med. 2016; 193: 1198-200.

13. Broos CE, Hendriks RW, Kool M. T-cell immunology in sarcoidosis: Disruption of a delicate balance between helper and regulatory T-cells. Curr Opin Pulm Med. 2016; 22: 476-83.

14. Costabel U, Guzman J. Bronchoalveolar lavage in interstitial lung disease. Curr Opin Pulm Med. 2001; 7: 255-61.

15. Ten Berge B, Paats MS, Bergen IM, et al. Increased IL-17A expression in granulomas and in circulating memory $\mathrm{T}$ cells in sarcoidosis. Rheumatology. 2012; 51: 37-46.

16. Mortaz E, Rezayat F, Amani D, et al. The Roles of T Helper 1, T Helper 17 and Regulatory T Cells in the Pathogenesis of Sarcoidosis. Iran J Allergy Asthma Immunol. 2016; 15: 334-9. 
17. Taflin C, Miyara M, Nochy D, et al. FoxP3+ regulatory $\mathrm{T}$ cells suppress early stages of granuloma formation but have little impact on sarcoidosis lesions. Am J Pathol. 2009; 174: 497-508

18. Rappl G, Pabst S, Riemann D, et al. Regulatory T cells with reduced repressor capacities are extensively amplified in pulmonary sarcoid lesions and sustain granuloma formation. Clin Immunol. 2011; 140: 71-83.

19. Oswald-Richter KA, Richmond BW, Braun NA, et al. Reversal of global CD4+ subset dysfunction is associated with spontaneous clinical resolution of pulmonary sarcoidosis. J Immunol. 2013; 190: 5446-53.

20. Bettelli E, Carrier Y, Gao W, et al. Reciprocal developmental pathways for the generation of pathogenic effector TH17 and regulatory T cells. Nature. 2006; 441: 235-8.

21. Buckner JH. Mechanisms of impaired regulation by CD4(+)CD25(+)FOXP3(+) regulatory T cells in human autoimmune diseases. Nat Rev Immunol. 2010; 10: 849-59.

22. Kimura A, Kishimoto T. IL-6: regulator of Treg/Th17 balance. Eur J Immunol. 2010; 40: 1830-5.

23. Mangan PR, Harrington LE, O'Quinn DB, et al. Transforming growth factor-beta induces development of the $\mathrm{T}(\mathrm{H}) 17$ lineage. Nature. 2006; 441: 231-4.

24. Sauer S, Bruno L, Hertweck A, et al. T cell receptor signaling controls Foxp3 expression via PI3K, Akt, and mTOR. Proc Natl Acad Sci U S A. 2008; 105 : 7797-802.

25. Haxhinasto S, Mathis D, Benoist C. The AKT-mTOR axis regulates de novo differentiation of CD4+Foxp3+ cells. J Exp Med. 2008; 205: 565-74.

26. Kang J, Huddleston SJ, Fraser JM, et al. De novo induction of antigen-specific CD4+CD25+Foxp3+ regulatory $\mathrm{T}$ cells in vivo following systemic antigen administration accompanied by blockade of mTOR. J Leukoc Biol. 2008; 83: 1230-9.

27. Merkenschlager $\mathrm{M}$, von Boehmer H. PI3 kinase signalling blocks Foxp3 expression by sequestering Foxo factors. J Exp Med. 2010; 207: 1347-50.

28. Huynh A, DuPage M, Priyadharshini B, et al. Control of PI(3) kinase in Treg cells maintains homeostasis and lineage stability. Nat Immunol. 2015; 16: 188-96.

29. Zanin-Zhorov A, Lin J, Scher J, et al. Scaffold protein Disc large homolog 1 is required for T-cell receptor-induced activation of regulatory T-cell function. Proc Natl Acad Sci U S A. 2012; 109: 1625-30. 